\title{
A PLATAFORMA WIKI NO ACESSO À INFORMAÇÃO DE ARQUIVOS PESSOAIS E MEMÓRIAS DE MÉDICOS
}

\begin{abstract}
Resumo: Buscou-se estudar o acesso às informações contidas num sistema de Banco de Dados em plataforma colaborativa. A funcionalidade é fornecer informações sobre armazenamento de dados sobre a profissão, vida, obra, pensamento, arquivos pessoais e memórias de médicos de Portugal e do Brasil que vão para muito além da Medicina. Iniciando-se pela Bahia e estendendo-se a captura de informação aos médicos da Comunidade de Países de Língua Portuguesa. A Mediawiki apresenta-se como espaço aberto à inserção de dados pelos próprios médicos e pessoas afins, interessadas em sua atualização on-line. Objetivamos apresentar abordagens conceituais sobre arquivos pessoais, informação, tecnologia digital, paradigma pós-custodial, interdisciplinaridade, plataformas colaborativas e arquitetura da informação. Acompanha compreensão teórica e conceitual do que se espera de um ambiente colaborativo. Apresentamos a nova interface do sistema, com a migração do banco de dados MYSQL, chamado por código $P H P$ em página $H T M L$. A metodologia adotada foi a revisão de literatura e aplicação de processos experimentais. Como resultados temos o preenchimento de novos dados e na inclusão de médicos com a utilização da nova interface. Duas barreiras foram transpostas: uma refere-se à limitação geográfica superada pela internet e a outra que permite a ação dos usuários médicos e pessoas afins, na proposição quantitativa do preenchimento do sistema de informação de médicos e a sua fiabilidade. Consideramos que o acesso à informação pela internet foi positivo, pois encontramos uma proposição quantitativa de atualização do sistema, com poder ilimitado de dados e acessos capazes de superar as transposições tecnológicas desta sociedade em rede.
\end{abstract}

Palavras-chave: acervos documentais pessoais. Memórias de médicos. Sistema de informação de médicos. Médicos e a cultura.
Daniel J. B. C. Branco

Professor técnico-universitário, administrador logístico/consultor em TIC, Doutorando em Ciência da Informação pela Universidade

Federal da Bahia (UFBA)

danielbranco.ufba@gmail.com

Gustavo Alpoim de Santana Professor universitário, administrador/ consultor em marketing e TIC. Mestre em Ciência da Informação pela Universidade Federal da Bahia (UFBA) gustavoalpoim@gmail.com

Zeny Duarte

Professora titular da pela Universidade Federal da Bahia (UFBA), doutora em Letras pela UFBA, pós-doutora em Ciência da Informação pela universidade do Porto, Portugal. zenydu@gmail.com

\section{WIKI PLATFORM ACCESS TO PERSONAL FILES AND MEDICAL DOCTORS MEMORIES INFORMATION}

\begin{abstract}
We sought to study the access to information contained in a collaborative platform database system. The function is to provide information regarding the storage of data about the profession, life, work, thought, personal files and memories of doctors from Portugal and Brazil that go far beyond medicine. Starting with Bahia and extending the capture of information to doctors of the Community of Portuguese Speaking Countries. Mediawiki presents itself as an open space for data entry by doctors themselves and others interested in updating it online. We aim to present conceptuais approaches on personal archives, information, digital technology, postcustodial paradigm, interdisciplinarity, collaborative platforms and information architecture. The system tracks theoretical and conceptual understanding of what is expected from a collaborative environment. Introducing the new system interface, with the MYSQL database migration, called by PHP code in HTML page. The methodology adopted was the literature review and application of experimental processes. As a result we have the filling of new data and the inclusion of doctors with the use of the new interface. Two barriers have been overcome: one regarding the geographical limitation overcome by the internet and the other that allows the action of medical users and related persons, in the quantitative proposition of filling the medical information system and its reliability. We consider that the access to information through the internet was positive, because we found a quantitative proposition to update of the system, with unlimited data and access power capable of overcoming the technological transpositions of this network society.
\end{abstract}

Keywords: Personal archives. Memories of Medical Doctors. medical information system. 


\section{INTRODUÇÃO}

Tratamos aqui do estudo sobre o acesso à informação ao sistema informatizado em plataforma colaborativa. A função desse sistema é o armazenamento de dados acerca da vida, obra, pensamento e acervos documentais pessoais de médicos do Brasil e de Portugal que produziram para além da Medicina, pesquisa iniciada pela Bahia e Portugal e, posteriormente, a se estender a outros estados do Brasil e demais integrantes da Comunidade dos Países de Língua Portuguesa (CPLP). O modelo segue a plataforma colaborativa Mediawiki, como espaço aberto à inserção de dados, pelos próprios médicos e pessoas afins interessadas em sua atualização on-line. Apresentamos abordagens conceituais sobre arquivos pessoais, informação, tecnologia digital, paradigma pós-custodial, interdisciplinaridade, plataforma Web, plataformas colaborativas, Arquitetura da Informação (AI), wikimedia, entre outras.

O sistema é demonstrado em Mediawiki, acompanha a compreensão teórica e conceitual sobre acesso à informação e colaboração do usuário. Até chegar ao estágio atual, o mencionado sistema passou por algumas experimentações. A primeira versão, criada no ano de 2008, teve uma interface possível para a época, porém espartana e clássica para os padrões técnicos vigentes. Na segunda versão, elaborada em 2011, aprimorou-se a relação com o internauta através dos recursos webnode, com o sistema de banco de dados por trás desta nova interface em backoffice. No ano de 2014, já na terceira versão, surge o projeto piloto com a transferência de alguns dados inseridos no antigo sistema para uma nova plataforma Wiki experimental. Obtendo para esta versão, como referencial técnicoconceitual, a Websemântica, metadados, taxonomia e ontologia representados no seguinte trabalho hospedado em um site hospedado na Universidade Federal do Rio Grande do Sul (UFRGS). Atualmente o Banco de Dados (BD) adota uma nova nomenclatura e encontra-se migrado de Microsoft Structured Query Language (MS-SQL) para My Structured Query Language (MySQL), estando o BD hospedado em servidores da Superintendência de Tecnologia e Informação (STI) órgão responsável por toda a gestão tecnológica informacional da Universidade Federal da Bahia (UFBA). Já lançado nacional e internacionalmente, através de sua divulgação em portais de universidades conveniadas e apoiadoras do projeto Os médicos e a cultura em Portugal e na Bahia, assim como também nas nuvens (cloud). 
Após a experimentação de três versões de interface para Web, visando alcançar resultados mais compatíveis com a evolução dos sistemas de informação, a indagação foi: Qual a contribuição da plataforma Wiki e a sua renovada interface na divulgação do projeto "Os médicos e a cultura em Portugal e na Bahia"? Esta é a nossa problematização e para respondê-la testamos a hipótese de que a utilização de um recurso tecnológico atualizado, neste caso a Mediawiki, implica no rápido acesso à informação do sistema relativa ao projeto citado anteriormente, baseando-se na interativa colaboração de seus usuários.

Portanto, objetivamos apresentar a renovada interface do sistema, com a migração do banco de dados MySQL em plataforma definitiva Mediawiki e avaliar o acesso à informação contida no banco de dados do referenciado sistema através da plataforma colaborativa instalada.

As ações de produção e divulgação da informação foram otimizadas pela facilidade de comunicação oferecida pela internet. Com foco nas facilidades de acesso à informação oferecidas pela tecnologia digital, justifica-se este projeto como uma tentativa de evidenciar os benefícios da aplicação do acesso à informação através de ferramentas tecnológicas no que diz respeito aos acervos documentais e produções de médicos baianos e portugueses. Diante desse contexto, e por ser para nós um tema familiar, acreditamos que a importância deste trabalho se dá pela necessidade de potencializar o acesso à informação através de uma nova ferramenta tecnológica denominada plataforma Wiki.

\section{REVISÃO DE LITERATURA}

Para a execução das atividades investigativas referentes ao projeto Médicos e a Cultura foi criado à época na UFBA o Grupo de Ensino, Extensão e Pesquisa em Ciência da Informação e Ciência da Saúde (GEPCIS). Hoje em dia quem assume as atividades de investigação é o grupo de pesquisa Acervos Manuscriptológicos, Bibliográficos, Iconográficos, Etnográficos: Organização, Preservação e Interfaces das Tecnologias da Informação e Comunicação G-ACERVOS, que se encontra credenciado pelo Conselho Nacional de Desenvolvimento Científico e Tecnológico (CNPq), o qual também desenvolve estudos sobre informação e saúde.

Para apoiar ainda mais esse projeto, quatro marcos miliares se fizeram presentes possibilitando a sua transposição do mundo das ideias para o mundo real, que apresentamos 
a seguir:

1. Criação do "Memorial de Medicina da Faculdade de Medicina" (na década de 80).

2. Convite em 2004 para implementação do projeto "Resgate do acervo da Faculdade de Medicina da Bahia: preservação, historicidade e salvaguarda do acervo".

3. Criação do GEPCIS, em 2006, o qual foi homologado e aprovado pelas congregações do Instituto de Ciência da Informação (ICI) e da FMB.

4. Publicação do livro “Os Médicos e a Cultura em Portuga1 e na Bahia (1808-2012)" com participação de especialistas e pesquisadores de renome das áreas da CI e Medicina, além de médicos convidados à escrita de suas próprias memórias.

Para assegurarmos a importância desse projeto Médicos e a Cultura e sua intrínseca multi, inter e transdisciplinaridade, representada pelas relações cientificas (teóricas, práticas e conceituais) entre as áreas da Arquivologia, Ciência da Computação, Comunicação, Medicina e outras ciências tecnológicas, sociais e humanas, expomos a seguinte proposição explicativa do contexto da inter-relação existente entre a cultura e informação:

\footnotetext{
A proposta conceituadora de Bauman favorece, mais que a definição de Morin, a justaposição cultura e informação, conferindo substrato eminentemente cultural à 'Era da Informação' em que estamos e em que assistimos, ao processo sinuoso e rápido de interpenetração profunda e extensa do tecnológico com todos os níveis do humano e do social (DUARTE; SILVA, 2016, p. 29).
}

Identificados os médicos e as pessoas detentoras de informações e conjuntos documentais destes profissionais da medicina que produziram e legaram à sociedade representativa produção nas áreas da filosofia, literatura, política, artes e cultura de alguma maneira, disseminar esse arcabouço cultural para a sociedade e torna-lo parte de uma base de dados que será utilizada como fonte de pesquisa e informação, é função primordial do projeto "Os médicos e a cultura em Portugal e na Bahia" com a utilização de seus dois principais filões: o SISMÉDICOS e o MEDINFOR.

A partir de 2017 o SISMÉDICOS encontra-se migrado de MS-SQL (Microsoft) para MySQL (open source), estando o banco hospedado em servidores da Superintendência de 
Tecnologia e Informação (STI) ${ }^{1}$ órgão responsável por toda a gestão tecnológica informacional da Universidade Federal da Bahia (UFBA) no seguinte endereço:

Como citado anteriormente a pesquisa baseia-se no levantamento das manifestações literárias, artísticas, políticas e culturais, os respectivos autores e titulares dos acervos e armazenamento de dados acerca da vida, obra, pensamento e acervos documentais pessoais (ADP) de médicos do Brasil e de Portugal que produziram para além da Medicina, encontrando em Silva (2004, p. 6) respaldo para o objeto em estudo

No caso vertente dos Arquivos Familiares e Pessoais estamos perante um Sistemas de Informação organizado ou operatório, cujo pólo estruturante e dinamizador é uma entidade - Família e Pessoa, cada qual com estrutura própria e acção fixada sempre por objectivos diversos, uns perenes e outros mutáveis. Aplica-se, assim, a teoria sistémica devidamente adaptada à ontologia do fenómeno informacional e através de um modelo que passaremos a caracterizar nos seus traços essenciais.

Para Silva (2004, p. 7),

o vector orgânico-funcional que releva do contexto histórico onde se situa a acção geradora de fluxo informacional tem de ser modelizado com o máximo de rigor num instrumento específico - o quadro orgânico-funcional - e, paralelamente, é imperioso que surjam gradativamente vários tipos de instrumentos de pesquisa: desde o guia ao catálogo de documentos ou actos informacionais com uma poderosa panóplia de índices (antroponímico, cronológico, geográfico, ideográfico, etc.).

A função da base de dados anteriormente citada é o armazenamento de dados acerca da vida, obra, pensamento e arquivos pessoais de médicos do Brasil e de Portugal que produziram para além da Medicina, pesquisa iniciada pela Bahia e Portugal e, posteriormente, a se estender a captura das informações sobre médicos da Comunidade dos Países de Língua Portuguesa (CPLP) atendendo ao que Silva (2004, p. 24) expõe:

Qual a vantagem para o utilizador de que a documentação seja disposta por fases da evolução vital de um ser humano em articulação com as diversas actividades e cargos/empregos por ele assumidos (desde estudante a profissional em diferentes ramos ou sectores)? Com as novas TIC, o processo de digitalização instala-se e difunde-se permitindo a reprodução por imagem de documento a documento com efeitos práticos imediatos: evita a consulta no suporte original e dispensa uma catalogação minuciosa, além de afastar do cardápio a receita do inventário e das classificações na realidade mais temáticas que orgânico-funcionais até há pouco 'dogma' absoluto. O suporte digital parece sugerir, como apregoam os defensores desta solução tecnológica, uma resposta simples à questão posta: a vantagem para o utilizador é mostrar a informação textual, numérica ou gráfica/imagética que ele busca sem arrumações temáticas, nem orgânicas, ficando este exercício hermenêutico a cargo e da exclusiva responsabilidade de quem precisa e busca

\footnotetext{
$1<$ http://www.websismedicos.ufba.br $>$.
} 
informação. O máximo que é tolerado é criar grupos documentais, um misto de identificação tipológica e temática à guisa de séries artificiais.

Os médicos, embora também seja um aspecto comum a outros profissionais, vêm dispensando interesse às produções referentes às letras, artes, política, fillosofia e religião sendo, por isso, necessário prosseguir com o levantamento das manifestações literárias, artísticas, políticas e culturais, dos respectivos autores e titulares dos acervos, bem como o debate das causas e das vivências individuais de quantos teceram laços estreitos para além da ciência, deixando legado à cultura, esta, eminentemente próxima de toda e qualquer representação que reflete valores sociais e de memória.

No entanto, insistimos neste ponto crucial: "todo e qualquer ser humano nascido e criado em qualquer ponto do planeta Terra age para sobreviver e fixa objectivos legitimadores e estimuladores da acção, de acordo com os seus ciclos existenciais”. (SILVA, 2004, p. 24).

Para os usuários de arquivos pessoais, os documentos produzidos no âmbito da vida privada permitem uma análise multifacetária do produtor do arquivo e uma compreensão diversa da própria sociedade.

As Tecnologias da Informação e da Comunicação são ferramentas imprescindíveis na função da salvaguarda e transferência da informação transformada em conhecimento validado e compartilhado por meios digitais.

Podemos observar a possível condição da Ciência da Informação enquanto refém ou não da Ciência da Computação. Vejamos a síntese de Silva (2009, p. 123-124):

\begin{abstract}
Com a passagem do paradigma custodial e patrimonialista ao paradigma pós-custodial, informacional e científico, a preservação tornou-se mais complexa acompanhando a evolução tecnológica e, hoje, é com consternação que se assiste ao desaparecimento de milhares de registos, não por dia ou por hora, mas num segundo, devido a qualquer descuido ou até ao deliberado propósito de premir a tecla delete e apagar do suporte electrónico a informação aí contida. Quem pode assegurar as soluções convenientes para que os discos duros da microinformática, os discos de backup, as pen ou canetas digitais ou os $c d$-rom não percam a informação neles gravadas e possam ser 'lidos' por outros equipamentos de hardware, que um futuro próximo nos vai trazer? Os informáticos e não os cientistas da informação.
\end{abstract}

Identificados os médicos e as pessoas detentoras de informações e conjuntos documentais destes profissionais baianos e portugueses que produziram e legaram à sociedade representativa produção nas áreas da filosofia, literatura, política, artes e cultura de alguma maneira, é necessário disseminar esse arcabouço cultural para a sociedade e tornálo parte de uma base de dados acessível que será utilizada como fonte de pesquisa e informação. 
Apresentamos algumas vantagens de se colocar na Web as informações: acessibilidade, uniformidade, baixo custo, familiaridade e eficiência. Segundo Rezende (2010, p. 200) “[...] ela pode ser utilizada juntamente com a valorização do ser humano e da adaptação de seus sistemas de informação para captação, tratamento, distribuição, disseminação e troca de informação no ambiente Internet e seus recursos [...]”.

A rápida expansão da internet tem aberto inúmeras possibilidades para a implantação de serviços computacionais. A Web é atualmente, o principal veículo para a prestação destes serviços, permitindo atingir um número cada vez maior e mais diversificado de usuários. $\mathrm{O}$ aumento da oferta dos sistemas computacionais torna o usuário cada vez mais exigente, escolhendo aqueles que melhor resolvam os seus problemas. A interface de usuário é uma parte fundamental no sucesso de um sistema Web, pois é a responsável direta em fazer com que o usuário consiga realizar suas tarefas de maneira fácil, rápida e satisfatória.

Através desta ferramenta tecnológica colocou-se abaixo a barreira geográfica que até então impedia uma maior cobertura por parte dos entrevistadores, que partiam de uma plataforma Web centralizada em que digitadores alimentavam o banco com as informações recolhidas pelos mesmos. Desta forma a única interação era no momento da entrevista entre a pessoa que cedia as informações e a pessoa que registrava essas informações.

A definição pelo ambiente Web colaborativo, em detrimento à arquitetura cliente servidor em servidores locais deve-se ao fato da Web ter uma maior cobertura de público, localmente ou mundialmente, de língua portuguesa.

A internet, pela sua facilidade de comunicação oferecida, otimizou em muito as ações de produção e divulgação da informação. Com foco nessas facilidades de acesso à informação oferecidas pela tecnologia digital, evidenciamos os benefícios da aplicação do acesso à informação através dessa ferramenta tecnológica no que diz respeito aos acervos documentais e produções de médicos baianos e portugueses:

\footnotetext{
A influência das redes baseadas na Internet vai além do número de seus usuários: diz respeito também à qualidade do uso. Atividades econômicas, sociais, políticas, e culturais essenciais por todo o planeta estão sendo estruturadas pela Internet e em torno dela, como por outras redes de computadores. De fato, ser excluído dessas redes é sofrer uma das formas mais danosas de exclusão em nossa economia e em nossa cultura (CASTELLS, 2003, p. 8).
}

Os médicos e as pessoas detentoras de informações e conjuntos documentais destes profissionais da medicina que produziram e legaram à sociedade representativa produção nas áreas da filosofia, literatura, política, artes e cultura de alguma maneira, deixaram, deixam e 
deixarão essas trilhas para que possamos encontrar esse arcabouço cultural e mostrá-lo para a sociedade e torná-lo parte de uma base de dados que será utilizada como fonte de pesquisa e informação.

\section{METODOLOGIA}

Diferentemente do que ocorre com os outros delineamentos, como experimento e o levantamento, as etapas do estudo de caso não se dão numa sequência rígida. Seu planejamento tende a ser mais flexível e com frequência o que foi desenvolvido numa etapa determina alterações na seguinte (GIL, 2010, p. 117).

Por tratar-se de um tema de abrangência das ciências sociais, a abordagem da pesquisa científica a ser realizada será qualitativa. Costa e Costa (2001) afirmam que a qualitativa se preocupa com a realidade que não pode ser quantificada, trabalha com o subjetivo dos sujeitos (crenças, valores, atitudes, etc...) e pode trabalhar com dados, porém o tratamento não deve envolver estatística avançada.

Classificação da pesquisa apresentada, quanto à:
a) Natureza: aplicada
b) Objetivos: descritiva
c) Método: qualitativa (e quantitativa)
d) Procedimento: estudo de caso

Yin (2010, p. 41), assinala uma observação importante:

é que o método de estudo de caso não é apenas uma forma de 'pesquisa qualitativa',
mesmo que possa ser reconhecida entre a variedade de opções da pesquisa qualitativa
(por exemplo, Creswell, 2007). Alguma pesquisa de estudo de caso vai além de um
tipo de pesquisa qualitativa, usando uma mistura de evidência quantitativa e
qualitativa. Além disso, os estudos de caso não precisam sempre incluir a evidência
observacional direta e detalhada marcada pelas outras formas de 'pesquisa
qualitativa'.

Também para Yin (2010, p. 43) devemos considerar todos os métodos de modo inclusivo e pluralista como sendo partícipes de um repertório no qual a escolha é sua na realização da pesquisa referente a ciência social.

O estudo de caso é uma investigação empírica que I) investiga um fenômeno contemporâneo em profundidade e em seu contexto de vida real, especialmente quando II) os limites entre o fenômeno e o contexto não são claramente evidentes (YIN, 2010, p. 39).

Ainda de acordo com Yin (2010, p. 40), a investigação do estudo de caso I) enfrenta a situação tecnicamente diferenciada em que existirão muito mais variáveis de interesse do 
que pontos de dados, e como resultado II) conta com múltiplas fontes de evidência, com os dados precisando convergir de maneira triangular, e como outro resultado III) beneficia-se do desenvolvimento anterior das proposições teóricas para orientar a coleta e a análise de dados.

\section{CAMINHO PERCORRIDO NA PESQUISA}

O ponto de partida para iniciar os trabalhos sobre a situação do SISMEDICOS iniciouse pela conta de e-mail do grupo de pesquisa GEPIS: smpermanecer@gmail.com, formado por sua maioria por bolsistas do programa PERMANECER ${ }^{2}$ da UFBA.

Fazendo uma releitura dos 432 e-mails trocados nos quatro anos que limitam essa comunicação (2008 a 2012) percebemos que 391 e-mails fazem referência a digitação unitária das informações culturais dos médicos (que correspondem a 36,15\% dos dados inseridos no BD), e e-mails que referenciam mais de dois médicos por comunicação.

Com um número de 1.195 registros cadastrados no banco de dados SISMEDICOS e com um universo de pesquisa muito extenso, conforme dados de 2013 do Conselho Regional de Medicina do Estado da Bahia (CREMEB), na ordem de 18.299 médicos cadastrados ativamente na base, onde cerca de $60 \%$, isto é, cerca de 10.759 médicos atuam em Salvador, chegamos ao número de $11,10 \%$ de cobertura da pesquisa do projeto SIS Médicos e a Cultura somente levando em consideração os números da capital.

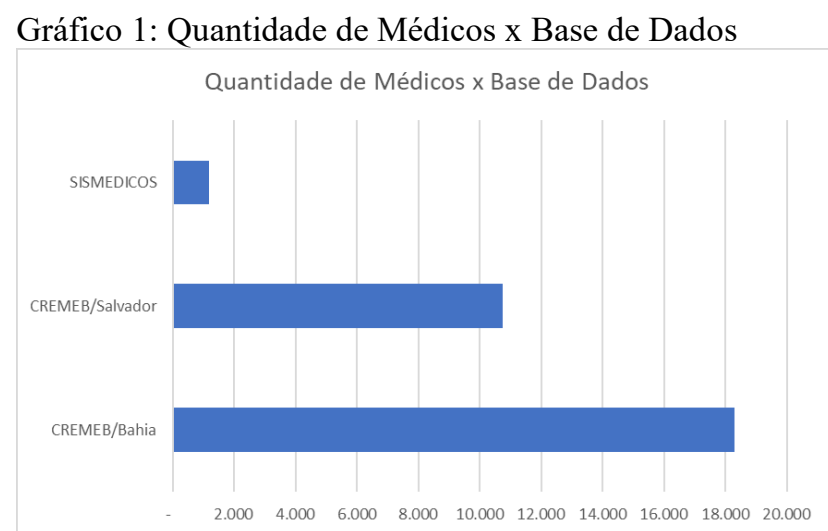

Fonte: elaborado pelo autor.

Levando em consideração os números apresentados no gráfico acima, avaliamos como muito promissora a empreitada deste projeto no âmbito do PPGCI/UFBA e G-

\footnotetext{
${ }^{2}$ Para maiores informações sobre o programa, acesse $<$ www.sisper.ufba.br $>>$
} 


\title{
5 ACESSO À INFORMAÇÃO
}

Buscando fazer um levantamento para “conhecer os hábitos de consumo de mídia da população brasileira a fim de subsidiar a elaboração da política de comunicação e divulgação social do Executivo Federal", a Secretaria de Comunicação Social da Presidência da República do Brasil encomendou para o IBOPE Inteligência um trabalho intitulado Pesquisa Brasileira de Mídia 2016 apresentando seu relatório final e dizendo resumidamente que

\begin{abstract}
[...] Entre os entrevistados, aproximadamente dois em cada três acessam a Internet; $\mathrm{o}$ ambiente domiciliar é predominante entre os locais de maior uso. $\mathrm{O}$ telefone celular supera e muito o computador como o dispositivo mais utilizado no acesso à Internet e algo em torno de três em cada dez respondentes que utilizam a Internet declaram utilizar somente um dispositivo para tal atividade. O tempo médio de acesso diário, considerando tanto o meio de semana quanto o final de semana, fica um pouco acima das quatro horas e trinta minutos [...]. (BRASIL, 2016, p. 33)
\end{abstract}

Isto quer dizer que aproximadamente $66 \%$ da população brasileira tem acesso à internet, confirmando o potencial de penetração do WEBSISMEDICOS na comunidade de usuários e reafirmando o papel da internet como ferramenta potencializadora de sua divulgação na comunidade global, buscando extrapolar os limites brasileiros e portugueses especificamente na abordagem dos demais países participantes da CPLP.

\section{COLABORAÇÃO PARTICIPATIVA DO USUÁRIO}

Em uma pesquisa estatística sobre os wikipedistas lusófonos (com 159 usuários), Alexandre Rosado em 2007 apresenta os seguintes dados: 2\% são usuários muito ativos, 17\% mais ou menos ativos, e 81 \% poucos ativos. Destes, 76,1\% são do Brasil e 16,4\% de Portugal. A maioria dos usuários são da região Sul e Sudeste do Brasil (com quase 40\%): em São Paulo (24,2\%) e Rio de Janeiro (15,4\%) (IMPRENSA/ENTREVISTA ISTOÉ, 2011).

A Wikipédia em língua portuguesa foi a quinta edição da Wikipédia a ser criada. Iniciou suas atividades em junho de 2001, tendo alcançado a marca de 460.000 artigos e mais de 70 administradores em 2011. Atualmente, é a oitava colocada em número de artigos no mundo e está no Brasil entre os 20 sítios mais visitados.

Então por analogia podemos projetar que o comportamento dos usuários para a 
produção de conteúdo em ambientes colaborativos é bastante promissor, principalmente no Brasil. Ambientes virtuais colaborativos ajudam a criar o conceito de Big Data. Chamamos de Big Data o conjunto de dados que se pode coletar, em grandessíssima quantidade, sendo possível a posterior análise, apenas usando os recursos da computação. Trabalhar com muitos dados sem a ajuda da computação pode ser complicado porque deixamos escapar informações importantes.

No nosso caso WEBSISMEDICOS o usuário pode colaborar na construção dos códigos das linguagens de programação e na alimentação dos dados referentes às expressões artístico culturais dos médicos, seja na criação dos dados ou mesmo na atualização deles.

Com o avanço dos conceitos da Web, os usuários poderão enriquecer ainda mais esse acervo artístico cultural adicionando imagens, sons, vídeos, textos e hiperlinks, enfim recursos multimídia às páginas wikis.

São os usuários produzindo conteúdo como atores sociais numa sociedade em rede, como preconiza Manuel Castells.

\section{CONSIDERAÇÕES FINAIS}

Considerando as atividades primordiais efetuadas por esta pesquisa, envolvendo análise de versões do banco de dados e suas interfaces expostas na internet, criação de um ambiente de colaboração visando a disseminação e democratização do acesso à informação, migração da base de dados para o novo ambiente, implementação de ferramentas auxiliares para a disseminação do sistema e demonstração de inconsistências referentes ao preenchimento da base de dados podemos avaliar como expressivas as exposições ora apresentadas.

O banco de dados se apresenta migrado para uma plataforma open source em todos os sentidos, desde o SGBD MySQL até o software colaborativo Mediawiki.

A escolha e a adoção de uma nova plataforma de acesso à informação, neste caso com colaboração participativa do usuário, ampliam a disseminação desta ferramenta dando oportunidade ao interessado de participar ativamente da construção deste arcabouço informacional.

A internet sendo um recurso tecnológico de alta necessidade na sociedade moderna contribuirá de forma expressiva para a rápida disseminação do sistema e consequentemente sua proposta de acesso democrático e universal à informação. 
A nova interface, mais dinâmica e clean, permite ao usuário ter acesso a ferramentas úteis para sua pesquisa, além de franquear a acessibilidade nas mais diversas plataformas de dispositivos: smartphones, tablets, netbooks, notebooks e desktops. O acesso ao sítio do sistema por meio dos dispositivos mencionados dá ao usuário maior independência nos questões espaço-temporais: o acesso as informações relativas aos médicos podem ser feitas em qualquer tempo e em qualquer lugar.

Assim posto, podemos constatar que os objetivos geral e específicos desta pesquisa foram atendidos, em relação à: I) apresentação da nova interface do sistema, agora sob nova denominação, com a migração do banco de dados MySQL em plataforma definitiva Wikimedia; II) avaliação do acesso à informação contida no banco de dados através da plataforma colaborativa instalada; III) verificação da integridade das informações inseridas até o presente momento no banco de dados; IV) apresentação das inconsistências encontradas dos dados inseridos; V) avaliação de como a informação estará disponível aos usuários; e VI) descrição das ferramentas interativas poderão ser usadas para a localização da informação por parte do usuário. Toda esta trajetória não poderia ter sido concretizada se não fosse nossa busca pelo aprofundamento teórico - conceitual sobre a área em estudo, como também a determinante participação em estudos interdisciplinares que sedimentaram nosso conhecimento em Ciência da Informação, aliado aos conhecimentos das Tecnologias da Informação e Comunicação.

Tivemos a preocupação de definir um percurso textual para situar o leitor na leitura deste trabalho, abordamos o Colóquio Internacional MEDINFOR “A Medicina na Era da Informação" e suas edições e a plataforma digital SISMÉDICOS e a Cultura na sua natural evolução técnica através das suas versões, dois produtos frutos do estudo pós-doutoral intitulado Os médicos e a cultura: estudo crítico e guia geral dos arquivos de médicos escritores, artistas e pensadores de Portugal e Bahia - Brasil (1808 - 2012). Citamos também os diversos trabalhos refletidos em artigos e livros que esse tema permitiu.

Através da análise do sistema exploramos a nova versão da plataforma colaborativa e assinalamos algumas características inovadoras tais como o acesso por meio de outras plataformas: smartphone, tablet e netbook.

A partir do atual estágio desta pesquisa, duas barreiras foram transpostas:

$1^{\mathrm{a}}$. Barreira - refere-se à limitação geográfica superada pela internet, que esperamos transpor ainda mais com a alcance do sítio na CPLP.

$2^{\mathrm{a}}$. Barreira - o sistema atual permite a ação do usuário, de forma colaborativa, nesse 
caso, médicos e pessoas afins, ampliando a proposição quantitativa do preenchimento do sistema e a sua fiabilidade, não mais dependendo da ação de um entrevistador, fazendo assim do usuário médico e pessoas afins um ator e/ou atriz social na construção deste arcabouço cultural.

Deixamos a proposta de uma maior interdisciplinaridade da CI com outras áreas, principalmente com a Ciência da Computação para a elaboração de um plano de ação com mudanças em relação a coleta de dados e especificações de campos e tipos de dados que, a partir deste estudo, alimentam o ambiente Mediawiki ao qual está inserido o projeto Médicos e a Cultura.

Também deixamos registrada a necessidade de uma revisão metodológica de todo o banco de dados e páginas wikis para efeitos de integridade das consultas a serem efetuadas pelos usuários, para não comprometerem a qualidade dos resultados das mesmas.

Como resultado, a utilização da plataforma colaborativa Mediawiki destaca-se no preenchimento de novos dados e na inclusão de médicos que ainda não estão no sistema.

Positivamente, foi encontrada uma proposição quantitativa de atualização do sistema, com poder ilimitado de dados e acessos capazes de superar as transposições tecnológicas desta sociedade da internet.

O projeto é extenso e permite a MIT dos conceitos da Ciência da Informação, neste caso representada pela Arquivologia e pela Ciência da Computação na solução das questões de suporte, recuperação e universalização do acesso à informação nesta era pós-custodial.

\section{REFERÊNCIAS}

BRASIL. Secretaria de Comunicação Social. Pesquisa brasileira de mídia - 2016: relatório final. Brasília, DF: Secretaria de Comunicação Social, 2016. Disponível em: $\mathrm{http}: / / w w w . s e c o m . g o v . b r / a t u a c a o / p e s q u i s a / l i s t a-d e-p e s q u i s a s-q u a n t i t a t i v a s-e-q u a l i t a t i v a s-$ de-contratos-atuais/pesquisa-brasileira-de-midia-pbm-2016.pdf/view

CASTELLS, M. A galáxia da internet: reflexões sobre a internet, os negócios e a sociedade. Rio de Janeiro: Zahar, 2003.

COSTA, M. A. F.; COSTA, M. de F. B. Metodologia de pesquisa: conceitos e técnicas. Rio de Janeiro: Interciência, 2001.

DUARTE, Z.; SILVA, A. M. da. Os médicos e a cultura em Portugal e na Bahia: olhar(es) 
introspectivo e analítico sobre o "modo de ser e estar" médico- cultural. Salvador: Edufba, 2016.

GIL, A. C. Como elaborar projetos de pesquisa. 5. ed. São Paulo: Atlas, 2010.

IMPRENSA/ENTREVISTA ISTOÉ. In: Wikimedia. 2011. Disponível em: https://br.wikimedia.org/wiki/Imprensa/Entrevista Isto\%C3\%89

REZENDE, D. A. Tecnologia da informação aplicada a sistemas de informação empresariais: o papel estratégico da informação e dos sistemas de informação nas empresas. 7. ed. São Paulo: Atlas, 2010.

SILVA, A. M. Arquivos familiares e pessoais: bases científicas para aplicação do modelo sistémico e interactivo. Revista da Faculdade de Letras, Ciências e Técnicas de Património, Porto, v. 3 p. 55-84, 2004. Disponível em: http://repositorioaberto.up.pt/bitstream/10216/8111/2/4083.pdf

SILVA, A. M. et al. Arquivística: teoria e prática de uma ciência da informação. 3. ed. Porto: Edições Afrontamentos, 2009.

SISMED. 2016. Disponível em: http:/www.ufrgs.br/sismed/wiki/index.php/P\%C3\%Algina principal. Acesso em: 15 jul 2019.

WEBSISMEDICOS. 2017. Disponível em: http://www.websismedicos.ufba.br/. Acesso em: 15 jul. 2019.

YIN, R. K. Estudo de caso: planejamento e métodos. 4. ed. Porto Alegre: Bookman, 2010. 